\title{
On the Optimality of Regularization Methods for Solving Linear Ill-Posed Problems
}

\author{
T. Schröter and U. Tautenhahn
}

\begin{abstract}
In this paper we consider a general class of regularization methods for ill-posed problems $A x=y$ where $A: X \rightarrow Y$ is a linear operator between Hilbert spaces $X$ and $Y$. The regularization methods have the general form $x_{\alpha}^{\delta}=\bar{x}+g_{a}\left(\left(A^{*} A\right)^{\circ+1}\right)\left(A^{*} A\right)^{*} A^{*}\left(y^{\delta}-A \bar{x}\right)$ where $y^{\delta}$ are the available noisy data with $\left\|y-y^{\delta}\right\| \leq \delta$. Assuming $x \in M_{p, E}=\{x \in X$ : $\left.x-\bar{x}=\left(A^{*} A\right)^{p / 2} v,\|v\| \leq E, p>0\right\}$ we consider different functions $g_{\alpha}$ and discuss the question how to choose the order $s$ and the regularization parameter $\alpha=\alpha(\delta, E, p)$ in order to obtain optimal estimates sup $\left\|x_{\alpha}^{\delta}-x\right\| \leq E^{1 /(p+1)} \delta^{p /(p+1)}$ where the supremum is taken over $x, \in M_{p, E}, y^{\delta} \in Y$ and $\left\|A x-y^{\delta}\right\| \leq \delta$.
\end{abstract}

Keywords: $I l l$-posed problems, regularization methods, optimality

AMS subject classiflcation: Primary $65 \mathrm{~J} 20$, secondary 47 A50

\section{Introduction}

In this paper we consider ill-posed problems

$$
A x=y .
$$

where $A \in \mathcal{L}(X, Y)$ is a linear bounded operator between infinite dimensional Hilbert spaces $X$ and $Y$ with non-closed range $R(A)$ of $A$. We introduce the set

$$
M_{p, E}=\left\{\dot{x} \in X: x-\bar{x}=\left(A^{*} A\right)^{p / 2} v,\|v\| \leq E, p>0\right\}
$$

where $\bar{x}$ can be considered as a suitable initial approximation for problem (1.1). We suppose that instead of $y$ noisy data $y^{\delta} \in Y$ are available and assume throughout this paper that

(A1) $\left\|y-y^{\delta}\right\| \leq \delta$
(A2) $x \in M_{p}, E$.

In different papers (cf., e.g., $[1,6,13])$ approximate solutions $x_{\alpha}^{\delta}$ for problem (1.1) are obtained according to

$$
x_{\alpha}^{\delta}=\bar{x}+g_{\alpha}\left(A^{*} A\right) A^{*}\left[y^{\delta}-A \bar{x}\right]
$$

T. Schröter: Techn. Univ. Chemnitz - Zwickau, Dep. Math., P.O. Box 964, D-09009 Chemnitz U. Tautenhahn: Hochsch. Techn., Wirtsch. u. Sozialwesen (BTWS) Zittau/Görlitz, Dep. Math., P.O. Box 261, D-02763 Zittau 
Here $g_{\alpha}(\lambda):[0, a] \rightarrow \mathbb{R}$ is a family of continuous functions depending on a positive regularization parameter $\alpha>0$ and $g_{\alpha}\left(A^{*} A\right)$ is defined according to $g_{\alpha}\left(A^{*} A\right)=\int_{0}^{a} g_{\alpha}(\lambda) d E_{\lambda}$ where $A^{*} A=\int_{0}^{a} \lambda d E_{\lambda}$ is the spectral decomposition of $A^{*} A$ and $a$ is a constant with $\left\|A^{*} A\right\| \leq a$. We introduce the worst case error $\Delta(\delta)$. for identifying $x$ from $y^{\delta}$ under the assumptions (A1), (A2) and the best possible error bound $\omega(\delta)$ according to

$$
\Delta(\delta)=\sup _{\substack{x \in M_{p, E}, y^{6} \in Y \\\left\|A x-y^{\delta}\right\| \leq \delta}}\left\|x_{\alpha}^{\delta}-x\right\| \text { and } \omega(\delta)=E^{1 /(p+1)} \delta^{p /(p+1)} .
$$

Then the regularization methods (1.2) are called

(i) order optimal on the set $M_{p, E}$ for a given parameter choice $\alpha=\alpha(\delta)$ if $\Delta(\delta) \leq$ $c \omega(\delta)$ with $c \geq 1$ holds,

(ii) optimal on the set $M_{p, E}$ for a given parameter choice $\alpha=\alpha(\delta)$ if $\Delta(\delta) \leq \omega(\delta)$ holds and

(iii) asymptotically optimal on the set $M_{p, E}$ for a given parameter choice $\alpha=\alpha(\delta)$ if $\overline{\lim }_{\delta \rightarrow 0} \Delta(\delta) / \omega(\delta)=1$.

For a general discussion of optimality and order optimality of the regularization methods (1.2) we refer to $[6,13,14]$.

In this paper we consider more general regularization methods. We introduce a family of methods according to

$$
x_{\alpha}^{\delta}=\bar{x}+g_{\alpha}\left(\left(A^{*} A\right)^{s+1}\right)\left(A^{*} A\right)^{s} A^{*}\left[y^{\delta}-A \bar{x}\right]
$$

depending on an additional parameter $s \geq-1 / 2$, where in case of negative $s$-values, $\left(A^{*} A\right)^{s}$ denotes the Moore-Penrose inverse of $\left(A^{*} A\right)^{-s}$. In the application of (1.4) one has to make different decisions. First one has to fix the method, i.e. one has to choose the function $g_{\alpha}:\left[0,\left\|A^{*} A\right\|^{s+1}\right] \rightarrow \mathbb{R}$, second one has to fix the order $s$, and third one has to choose a suitable value for the regularization parameter $\alpha$. In this paper we consider different functions $g_{\alpha}$ and want to find out for which values of $s$ there holds $\Delta(\delta)=\omega(\delta)$ provided $\alpha$ is chosen properly. Since we are interested in regularization methods (1.4) that are optimal on the set $M_{p, E}$, for the correct choice of the regularization parameter $\alpha$ information about $\delta, E$ and $p$ will be necessary. Optimality results for special regularization methods have been known before. In the special case $s=0$ it is known that

(i) the method of Tikhonov regularization (cf. Section 3) is optimal for $0<p \leq 2$ if $\alpha$ is chosen by $\alpha=\frac{1}{p}\left(\frac{\delta}{E}\right)^{2 /(p+1)}$ (cf. [14]),

(ii) the method of regularized singular value decomposition (cf. Section 4) is optimal for all $p>0$ if $\alpha$ is chosen by $\alpha=\left(\frac{p+1}{p}\right)^{1 / \tau}\left(\frac{\delta}{E}\right)^{2 /(p+1)}$ (cf. [9] for the case $\tau=1 / 2$ and [11] for the case $\tau=1$ ),

(iii) the method of regularized singular value decomposition (1.2) with the function $g_{\alpha}(\lambda)=1 / \lambda$ for $\lambda \geq \alpha$ and $g_{\alpha}(\lambda)=0$ for $\lambda<\alpha$ is not optimal for any parameter choice $\alpha=\alpha(\delta)$ (cf. [6]),

(iv) the method of asymptotical regularization (cf. Section 5) is optimal for $0<$ $p \lesssim 7.124$ if $\alpha$ is chosen by $\alpha=\frac{1}{\ln (p+1)}\left(\frac{\delta}{E}\right)^{2 /(p+1)}$ (cf. [14]) and 
(v) the Landweber iteration method (cf. Section 7) is asymptotically optimal for $0<p \lesssim 7.124$ if the iteration number $N$ is chosen as the integer part of $1 / \alpha$ with $\alpha$ chosen as in the method of asymptotical regularization (cf. [14]).

In the case $s \neq 0$ some optimality results are known for the method of Tikhonov regularization (cf. [6]) and for the method of regularized singular value decomposition (cf. [11]). For some further optimality results and non-optimality results in case $s=0$ we refer to $[5,6,9,11,13-15]$. A posteriori parameter choice strategies that yield order optimal error bounds (1.3) without to know $E$ and $p$ are discussed, e.g., in $[2,11,13]$.

From the above discussion of optimality results for the special case $s=0$ we realize that the regularization methods (1.2) are optimal for $p \in\left(p_{0}, p_{1}\right]$ with $p_{0}=0$, where the upper bound $p_{1}$ depends on the special choice of the function $g_{\alpha}$. Hence, there appears a saturation effect: If assumption (A2) (which can be considered as a given a priori information on $x-\bar{x}$ ) is satisfied with $p>p_{1}$, then it is impossible to find any parameter choice $\alpha=\alpha(\delta)$ such that the regularization method is optimal on $M_{p, E}$. An advantage of the regularization scheme (1.4) over (1.2) is that optimality can be guaranteed if the parameter $s$ is chosen appropriately, consequently, saturation effects can be prevented by the right choice of $s$. Roughly speaking we can say, the larger $s$ is chosen, the larger the upper and lower bounds $p_{1}$ and $p_{0}$ (compare Figures 1, 3 and 4 and note that the real numbers $(s, p)$ and $(\kappa, \mu)$ are related by (2.4)). On the other hand, if $s$ has been chosen too large (such that (A2) holds with $p<p_{0}$ ), then again optimality cannot be guaranteed. Some other situation we can observe for the method of regularized singular value decomposition discussed in Section 4 (which depends on an additional parameter $\tau>0$ ). In this method optimality can be guaranteed for all $p \in(0, \infty)$ provided $\tau$ and $s$ are related by $\tau \leq 1 /(1+s) \leq 2 \tau$.

\section{Optimality examinations}

Let $\Delta(\delta)$ the maximal error defined in (1.3) and $\sigma\left(A^{*} A\right)$ the spectrum of the operator $A^{*} A$. Then we find from Lemma 2.2 in [14] the following

Lemma 2.1. Let $A \in \mathcal{L}(X, Y)$. Then for $x_{\alpha}^{\delta}$ defined in (1.4) there.holds the representation

$$
\begin{gathered}
\Delta(\delta)=\inf _{0<\xi<1} \| \frac{E^{2}}{\xi}\left(A^{*} A\right)^{p}\left[I-\left(A^{*} A\right)^{s+1} g_{\alpha}\left(\left(A^{*} A\right)^{s+1}\right)\right]^{2} \\
+\frac{\delta^{2}}{1-\xi}\left(A^{*} A\right)^{2 s+1} g_{\alpha}^{2}\left(\left(A^{*} A\right)^{s+1}\right) \|^{1 / 2}
\end{gathered}
$$

For $0 \leq t<\infty$ and $0<\alpha<\infty$ we introduce the funçtions $g:[0, \infty) \rightarrow \mathbb{R}$ and $h:[0, \infty) \rightarrow \mathbb{R}$ according to

$$
g(t)=\alpha g_{\alpha}(\alpha t) \quad \text { and } \quad h(t)=1-t g(t)
$$

and assume that they are independent on $\alpha$. We apply Lemma 2.1 and obtain 
Lemma 2.2. Let $A \in \mathcal{L}(X, Y)$ and let (2.1) hold. Then, for $x_{\alpha}^{\delta}$ defined in (1.4) with $\alpha=k\left(\frac{\delta}{E}\right)^{(2 s+2) /(p+1)}, k>0$, there holds the representation

$$
\Delta(\delta)=\inf _{0<\xi<1} \sup \left\{\frac{(k y)^{p /(s+1)} h^{2}(y)}{\xi}+\frac{(k y)^{-1 /(s+1)} y^{2} g^{2}(y)}{1-\xi}\right\}^{1 / 2} E^{\frac{1}{p+1}} \delta^{\frac{p}{p+1}}
$$

where the supremum is taken over $k y \in \sigma\left(\left(\frac{\delta}{E}\right)^{-(2 s+2) /(p+1)}\left(A^{*} A\right)^{s+1}\right)$.

Proof. From Lemma 2.1 we obtain with the substitution $t^{s+1}=\alpha y$ and (2.1) that

$$
\begin{aligned}
\Delta(\delta)=\inf _{0<\xi<1} \sup _{t \in \sigma\left(A^{*} A\right)}\left\{\frac{E^{2}}{\xi} t^{p}\left[1-t^{s+1} g_{\alpha}\left(t^{s+1}\right)\right]^{2}+\frac{\delta^{2}}{1-\xi} t^{2 s+1} g_{\alpha}^{2}\left(t^{s+1}\right)\right\}^{1 / 2} \\
=\inf _{0<\xi<1} \sup _{\alpha y \in \sigma\left(\left(A^{*} A\right)^{*+1}\right)}\left\{\frac{E^{2}}{\xi}(\alpha y)^{\frac{p}{s+1}}[1-y g(y)]^{2}\right. \\
\left.+\frac{\delta^{2}}{1-\xi}(\alpha y)^{\frac{-1}{s+1}} y^{2} g^{2}(y)\right\}^{1 / 2}
\end{aligned}
$$

We use the parameter choice of Lemma 2.2 and obtain the desired result (2.2)

Now one can show that $(\xi, k)=\left(\frac{1}{p+1}, \frac{1}{h^{-1}(1 /(p+1))}\right)$ is a stationary point of the expression $\{\ldots\}$ in (2.2) as a function of $\xi$ and $k$. We substitute this point into (2.2), introduce the real numbers

$$
\kappa=\frac{1}{1+s} \quad \text { and } \quad \mu=\frac{p}{1+s}
$$

and obtain the following

Theorem 2.3. Let $A \in \mathcal{L}(X, Y)$ and let (2.1) and (2.4) hold. If

(i) the equation $h(t)=\frac{1}{p+1}$ has a unique solution $t_{0}$

(ii) $\alpha$ is chosen by $\alpha=\frac{1}{t_{0}}\left(\frac{\delta}{E}\right)^{(2 s+2) /(p+1)}$

then for $x_{\alpha}^{\delta}$ defined in (1.4) there holds the error estimate

$$
\Delta(\delta) \leq E^{\frac{1}{p+1}} \delta^{\frac{p}{p+1}} \sup _{0 \leq y<\infty} f(y)
$$

with

$$
f(y)=\left\{\frac{\kappa+\mu}{\kappa}\left(\frac{y}{t_{0}}\right)^{\mu} h^{2}(y)+\frac{\kappa+\mu}{\mu}\left(\frac{y}{t_{0}}\right)^{-\kappa} y^{2} g^{2}(y)\right\}^{1 / 2} .
$$


Remark 2.4. Under the conditions of Theorem 2.3 the regularization method't 1.4 ), (2.1) is optimal on $M_{p, E}$ if $f(y) \leq 1$ holds for all $y \geq 0$. If $f(y)>1$ for some $y \in[0, \infty)$ and $\sigma\left(A^{*} A\right) \supset[0, \varepsilon], \varepsilon>0$, then the method (1.4) is not optimal on $M_{p, E}$ for any parameter choice $\alpha=\alpha(\delta)$, provided $\delta=\delta(\varepsilon)$ is sufficiently small. For the proof of some non-optimality results we refer to $[6,14,15]$.

Remark 2.5. From (2.2) we obtain that the method (1.4) is order optimal on $M_{\mathrm{p}, E}$ for the parameter choice $\alpha=k\left(\frac{\delta}{E}\right)^{(2 s+2) /(p+1)}$ with a constant $k>0$ if both $\sup _{y \geq 0} y^{\mu} h^{2}(y)<\infty$ and $\sup _{y \geq 0} y^{2-\kappa} g^{2}(y)<\infty$ hold where $\mu$ and $\kappa$ are the constants from (2.4) and $g$ and $h$ are the functions from (2.1).

Remark 2.6. The results of this paper can also be used in order to study optimality questions with respect to the more general $\|\cdot\|_{r}$-norm, $r \in \mathbb{R}$, where $\|x\|_{r}=$ $\left\|\left(A^{*} A\right)^{-r / 2} x\right\|$, since in analogy to Theorem 2.3 there holds the following result : Let $A \in \mathcal{L}(X, Y)$ and let $(2.1), \kappa=(1+r) /(1+s)$ and $\mu=(p-r) /(1+s)$ hold. If

(i) the equation $h(t)=\frac{\kappa}{\kappa+\mu}$ has a unique solution $t_{0}$

(ii) $\alpha$ is chosen by $\alpha=\frac{1}{i_{0}}\left(\frac{\delta}{E}\right)^{2 /(\kappa+\mu)}$

then for $x_{\alpha}^{\delta}$ defined in (1.4) there holds the error estimate

$$
\sup _{\substack{x \in M_{p, E}, y^{6} \in Y \\\left\|A x-y^{6}\right\| \leq \delta}}\left\|x_{\alpha}^{\delta}-x\right\|_{r} \leq E^{\frac{\kappa}{\kappa+\mu}} \delta^{\frac{\mu}{\kappa+\mu}} \sup _{y \geq 0} f(y)
$$

where $f(y)$ is given by (2.6).

\section{Optimality of Tikhonov methods}

In these regularization methods the regularized solution $x_{\alpha}^{\delta}$ is obtained from (1.4) with $g_{\alpha}(t)=1 /(t+\alpha)$, hence, $x_{\alpha}^{\delta}$ is the solution of the operator equation

$$
\left[\left(A^{*} A\right)^{s+1}+\alpha I\right]\left(x_{\alpha}^{\delta}-\bar{x}\right)=\left(A^{*} A\right)^{s} A^{*}\left[y^{\delta}-A \bar{x}\right] .
$$

Consequently, for the functions $g$ and $h$ of (2.1) we have $g(t)=1 /(t+1)$ and $h(t)=$ $1 /(t+1)$. The equation $h(t)=1 /(p+1)$ has the unique solution $t_{0}=p$, hence from Theorem 2.3 we obtain

Corollary 3.1. Let $A \in \mathcal{L}(X, Y)$ and $x_{\alpha}^{\delta}$ the regularized solution of equation (3.1) where $\alpha$ is given by the a priori parameter choice $\alpha=\frac{1}{p}\left(\frac{6}{E}\right)^{(2 s+2) /(p+1)}$. If the constants $\kappa$ and $\mu$ from (2.4) satisfy the inequality

$$
f(y)=\frac{\kappa+\mu}{\kappa}\left(\frac{\kappa}{\mu}\right)^{\mu} \frac{y^{\mu}}{(y+1)^{2}}+\frac{\kappa+\mu}{\mu}\left(\frac{\mu}{\kappa}\right)^{\kappa} \frac{y^{2-\kappa}}{(y+1)^{2}} \leq 1
$$

for all $y \in[0, \infty)$, then there holds the error estimate

$$
\Delta(\delta) \leq E^{\frac{1}{p+1}} \delta^{\frac{p}{p+1}} .
$$


Theorem 3.2. Let $A \in \mathcal{L}(X, Y)$ and $x_{\alpha}^{\delta}$ the regularized solution of equation (3.1) where $\alpha$ is given by the a priori parameter choice strategy $\alpha=\frac{1}{p}\left(\frac{\delta}{E}\right)^{(2 s+2) /(p+1)}$. If the constants $\kappa$ and $\mu$ from (2.4) satisfy the inequalities

$$
\begin{array}{ll}
0<\kappa \leq 2, & 0<\mu \leq 2 \\
\kappa+\mu \geq 1, & \mu^{2} \kappa+\kappa^{2} \mu \leq \kappa^{2}+\kappa \mu+\mu^{2}
\end{array}
$$

then there holds the error estimate (3.3).

Proof. We apply Corollary 3.1 and prove that (3.2) follows from (3.4). The first two inequalities of (3.4) guarantee that $\sup _{0 \leq y<\infty} f(y)<\infty$ holds. We transform (3.2) into the equivalent inequality

$$
g(y):=-y^{2}+\frac{\kappa+\mu}{\kappa}\left(\frac{\kappa}{\mu}\right)^{\mu} y^{\mu}+\frac{\kappa+\mu}{\mu}\left(\frac{\mu}{\kappa}\right)^{\kappa} y^{2-\kappa}-2 y \leq 1
$$

and decompose $g(y)$ into the sum $g_{1}(y)+g_{2}(y)$ with

$$
\begin{aligned}
& g_{1}(y)=-a y^{2}+\frac{\kappa+\mu}{\kappa}\left(\frac{\kappa}{\mu}\right)^{\mu} y^{\mu}-b y \\
& g_{2}(y)=(a-1) y^{2}+\frac{\kappa+\mu}{\mu}\left(\frac{\mu}{\kappa}\right)^{\kappa} y^{2-\kappa}+(b-2) y
\end{aligned}
$$

and $a=\frac{\kappa}{2 \mu}[\kappa+\mu-b]$, where $b$ is an arbitrary constant. We observe that

(i) $g_{1}^{\prime}\left(\frac{\mu}{\kappa}\right)=0$ and $g_{2}^{\prime}\left(\begin{array}{c}\mu \\ \kappa\end{array}\right)=0$,

(ii) $\lim _{y \rightarrow \infty} g_{1}(y)=-\infty$ and $\lim _{y \rightarrow \infty} g_{2}(y)=-\infty$ if $0<a<1$, i.e.

$$
\mu+\kappa-\frac{2 \mu}{\kappa}<b<\mu+\kappa
$$

(iii) $g_{1}\left(\frac{\mu}{\kappa}\right) \geq 0$ and $g_{2}\left(\begin{array}{l}\mu \\ \kappa\end{array}\right) \geq 0$ if and only if

$$
2-\mu-\kappa \leq b \leq \frac{(2-\mu)(\mu+\kappa)}{\mu} .
$$

Now we conclude as follows: From the last two inequalities of (3.4) we have

$$
2-\mu-\kappa<\mu+\kappa \quad \text { and } \quad \mu+\kappa-\frac{2 \mu}{\kappa}<\frac{(2-\mu)(\kappa+\mu)}{\mu} \text {, }
$$

hence there exists a constant $b$ such that (3.5) and (3.6) hold. Consequently, from (i) (iii) there follows

$$
\sup _{y \geq 0} g_{1}(y)=g_{1}\left(\begin{array}{l}
\mu \\
\kappa
\end{array}\right) \cdot \quad \text { and } \quad \sup _{y \geq 0} g_{2}(\dot{y}) \doteq g_{2}\left(\begin{array}{l}
\mu \\
\kappa
\end{array}\right)
$$

which gives $\sup _{y \geq 0} g(y) \leq g_{1}\left(\frac{\mu}{\kappa}\right)+g_{2}\left(\frac{\mu}{\kappa}\right)=1$. Hence, the proof is complete 
Remark 3.3. Theorem 3.2 shows that the method (3.1) is optimal on $M_{p, E}$ for a special parameter choice if the pair $(\kappa, \mu)$ belongs to the range defined by (3.4). In order to check if there are further $(\kappa, \mu)$-values for which the method (3.1) is optimal we have examined the function (3.2) numerically. We have found that also for $(\kappa, \mu)$-values of the range $B$ in Figure 1 there holds $\sup _{y \geq 0} f(y) \leq 1$.

Remark 3.4. Let $(\kappa, \mu) \in(0,2] \times(0,2)$. Then the method (3.1) is order optimal on $M_{p, E}$ for the parameter choice $\alpha=k\left(\frac{\delta}{E}\right)^{(2 s+2) /(p+1)}$ with a constant $k>0$. The proof of this result follows from Remark 2.5. The $(\kappa, \mu)$-range in which the method (3.1) is optimal or order optimal is given in Figure 1.

Figure 1. $(\kappa, \mu)$-range of optimality for method (3.1)

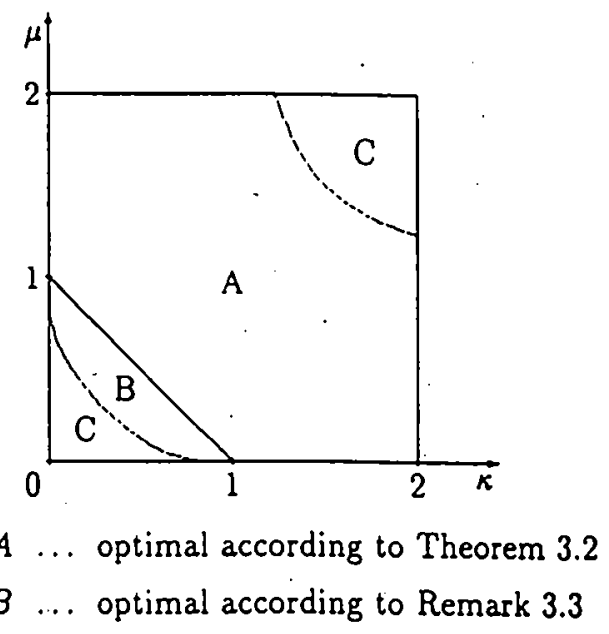

$A \cup B \cup C \ldots$ order optimal according to Remark 3.4

\section{Optimality of singular value decomposition methods}

In the generalized methods of regularized singular value decomposition the regularized solution $x_{\alpha}^{\delta}$ is obtained from (1.4) with

$$
g_{\alpha}(t)= \begin{cases}1 / t & \text { for } t \geq \alpha \\ (1 / \alpha)(\alpha / t)^{1-r} & \text { for } t<\alpha\end{cases}
$$

with $\tau \in(0, \infty)$, consequently, for the functions $g$ and $h$ of $(2.1)$ we have

$$
g(t)=\left\{\begin{array}{ll}
1 / t & \text { for } t \geq 1 \\
(1 / t)^{1-r} & \text { for } t<1
\end{array} \quad \text { and } \quad h(t)= \begin{cases}0 & \text { for } t \geq 1 \\
1-t^{r} & \text { for } t<1 .\end{cases}\right.
$$

The equation $h(t)=1 /(p+1)$ has the unique solution $t_{0}=(p /(p+1))^{1 / \tau}$, hence, from Theorem 2.3 we obtain 
Corollary 4.1. Let $A \in \mathcal{L}(X, Y)$ and $x_{\alpha}^{\delta}$ the regularized solution of equation (1.4) where $g_{\alpha}$ is the function from (4.1) and $\alpha$ is given by the a priori parameter choice $\alpha=\left(\frac{p+1}{p}\right)^{1 / \tau}\left(\frac{\delta}{E}\right)^{(2 s+2) /(p+1)}$. If the constants $\kappa$ and $\mu$ from (2.4) satisfy the inequality $\sup _{y \geq 0} f(y) \leq 1$ where

$$
f(y)= \begin{cases}\left(\frac{\kappa+\mu}{\mu}\right)^{1-\kappa / \tau} y^{-\kappa} & \text { for } y \geq 1 \\ \frac{\kappa+\mu}{\kappa}\left(\frac{\kappa+\mu}{\mu}\right)^{\mu / \tau} y^{\mu}\left(1-y^{\tau}\right)^{2}+\left(\frac{\mu}{\kappa+\mu}\right)^{\kappa / \tau-1} y^{2 \tau-\kappa} & \text { for } y<1\end{cases}
$$

then there holds the error estimate (3.3).

Theorem 4.2. Let $A \in \mathcal{L}(X, Y)$ and $x_{\alpha}^{\delta}$ the regularized solution of equation (1.4) where $g_{\alpha}$ is the function from (4.1) and $\alpha$ is given by the a priori parameter choice $\alpha=\left(\frac{p+1}{p}\right)^{1 / \tau}\left(\frac{\delta}{E}\right)^{(2 s+2) /(p+1)}$. If the constants $\kappa$ and $\mu$ from (2.4) satisfy the inequalities

$$
\tau \leq \kappa \leq 2 \tau \quad \text { and } \quad 0<\mu<\infty
$$

then there holds the error estimate (3.3).

Proof. We apply Corollary 4.1 and prove that $\sup _{y \geq 0} f(y) \leq 1$ holds if (4.3) is satisfied. First we note that $\sup _{y \geq 0} f(y)<\infty$ if $0<\kappa \leq 2 \tau$ and $0<\mu<\infty$ hold. Let $0<\kappa \leq 2 \tau$. Then $f(y)$ is monoton decreasing for $y \geq 1$. Furthermore, $f(1) \leq 1$ is satisfied for $\kappa \geq \tau$. Hence, it remains to prove that for $(\kappa, \mu) \in[\tau, 2 \tau] \times(0, \infty)$ there holds $\sup _{0 \leq y \leq 1} f(y) \leq 1$. For $0 \leq y^{\tau} \leq 1$ there holds the inequality

$$
\left(y^{\tau}\right)^{\mu}\left(1-y^{\tau}\right)^{\kappa} \leq\left(\frac{\mu}{\mu+\kappa}\right)^{\mu}\left(\frac{\dot{\kappa}}{\mu+\kappa}\right)^{\kappa}
$$

which gives

$$
y^{\mu} \leq\left(\frac{\mu}{\mu+\kappa}\right)^{\mu / \tau}\left(\frac{\kappa}{\mu+\kappa}\right)^{\kappa / \tau}\left(1-y^{\tau}\right)^{-\kappa / \tau} .
$$

We use this inequality and obtain from (4.2)

$$
f(y) \leq\left(\frac{\kappa}{\mu+\kappa}\right)^{\kappa / \tau-1}\left(1-y^{\tau}\right)^{2-\kappa / \tau}+\left(\frac{\mu}{\mu+\kappa}\right)^{\kappa / \tau-1}\left(y^{\tau}\right)^{2-\kappa / \tau}
$$

for $0 \leq y \leq 1$. One shows that the right-hand side of this inequality attains its maximum for $y^{\top}=\mu /(\mu+\kappa)$ which yields the desired result $\sup _{0 \leq y \leq 1} f(y) \leq 1$ for $(\kappa, \mu) \in[\tau, 2 \tau] \times(0, \infty)$

Remark 4.3. Theorem 4.2 shows that the method (1.4) with (4.1) is optimal on $M_{p, E}$ for a special parameter choice if the pair $(\kappa, \mu)$ belongs to the range defined by (4.3). An analytical examination of the function (4.2) shows that there are no further $(\kappa, \mu)$-values satisfying $\sup _{y \geq 0} f(y) \leq 1$. 
Remark 4.4. Let $(\kappa, \mu) \in(0,2 \tau] \times(0, \infty)$. Then the method (1.4) with (4.1) is order optimal on $M_{p, E}$ for the parameter choice $\alpha=k\left(\frac{\delta}{E}\right)^{(2,+2) /(p+1)}$ with a constant $k>0$. The proof of this result follows from Remark 2.5. The $(\kappa, \mu)$-range in which the method (3.1) is optimal or order optimal is given in Figure 2.

Remark 4.5. We note that for operators with discrete spectrum the computation of (1.4), (4.1) can be done according to

$$
\begin{aligned}
x_{\alpha}^{\delta}=\bar{x} & +\sum_{s_{i}^{2 \rho+2} \geq \alpha} \frac{\left(z^{\delta}-A \bar{x}, v_{i}\right)}{s_{i}} u_{i} \\
& +\frac{1}{\alpha^{\tau}}\left\{\left(A^{*} A\right)^{\rho} A^{*}\left(z^{\delta}-A \bar{x}\right)-\sum_{\Delta_{i}^{2 \rho+2} \geq \alpha} s_{i}^{2 \rho+1}\left(z^{\delta}-A \bar{x}, v_{i}\right) u_{i}\right\}
\end{aligned}
$$

with $\rho=\tau(s+1)-1$ where $\left\{s_{i}, u_{i}, v_{i}\right\}_{i \in \mathbf{N}}$ denotes the singular system of $A \in \mathcal{L}(X, Y)$. Recommendations for $s$ and $\tau$ are values with $2 \tau(s+1)=1$ (e.g. $s=-1 / 2$ and $\tau=1$ ) since in this case (4.3) is satisfied for all $p>0$ and the number of summands to be computed in the above two sums is as small as possible supposed $\alpha$ is chosen by the optimal parameter choice of Theorem 4.2.

Figure 2. $(\kappa, \mu)$-range of optimality for method (1.4), (4.1)



A ... optimal according to Theorem 4.2

$A \cup B$... order optimal according to Remark 4.4

\section{Optimality of asymptotical regularization methods}

In these methods the regularized solution $x_{\alpha}^{\delta}$ is characterized by (1.4) with $g_{\alpha}(t)=$ $\left(1-e^{-t / \alpha}\right) / t$, hence, there holds $x_{\alpha}^{\delta}=y(T)$, where $y(t)$ is the solution of the initial value problem

$$
\begin{aligned}
\dot{y}(t)+\left(A^{*} A\right)^{s+1} y(t) & =\left(A^{*} A\right)^{*} A^{*} y^{6} \quad, \quad t \in(0, T] \\
y(0) & =\bar{x}
\end{aligned}
$$


and $\alpha=1 / T$ denotes the regularization parameter. For the functions $g$ and $h$ of $(2.1)$ we have $g(t)=\left(1-e^{-t}\right) / t$ and $h(t)=e^{-t}$. The equation $h(t)=1 /(p+1)$ possesses the unique solution $t_{0}=\ln (p+1)$, hence, from Theorem 2.3 there follows

Corollary 5.1. Let $A \in \mathcal{L}(X, Y)$ and $x_{\alpha}^{\delta}=y(T)$ where $y(t)$ is the solution of (5.1). Let $\alpha=1 / T$ be chosen according to $\alpha=\frac{1}{\ln (p+1)}\left(\frac{\delta}{E}\right)^{(2 s+2) /(p+1)}$. If the constants $\kappa$ and $\mu$ from (2.4) satisfy the inequality

$$
\begin{aligned}
f(y) & =\frac{\kappa+\mu}{\kappa}\left(\ln \frac{\kappa+\mu}{\kappa}\right)^{-\mu} y^{\mu} e^{-2 y}+\frac{\kappa+\mu}{\mu}\left(\ln \frac{\kappa+\mu}{\kappa}\right)^{\kappa} y^{-\kappa}\left(1-e^{-y}\right)^{2} \\
& \leq 1
\end{aligned}
$$

for all $y \in[0, \infty)$, then there holds the error estimate (3.3).

Remark 5.2. In order to check for which $(\kappa, \mu)$-values the generalized method of asymptotical regularization is optimal on $M_{p, E}$ we have examined the function $f(y)$ of Corollary 5.1 numerically. We have found that for $(\kappa, \mu)$-values of the range $A$ in Figure 3 there holds $\sup _{y \geq 0} f(y) \leq 1$, hence, in this range the method is optimal.

Remark 5.3. Let $(\kappa, \mu) \in(0,2] \times(0, \infty)$. Then the generalized method of asymptotical regularization is order optimal on $M_{p, E}$ for the parameter choice $\alpha=$ $k\left(\frac{\delta}{E}\right)^{(2 s+2) /(p+1)}$ with a constant $k>0$. The proof of this result follows from Remark 2.5. The $(\kappa, \mu)$-range in which this method is optimal or order optimal is given in Figure 3.

Figure 3. $(\kappa, \mu)$-range of optimality for the asymptotical regularization method

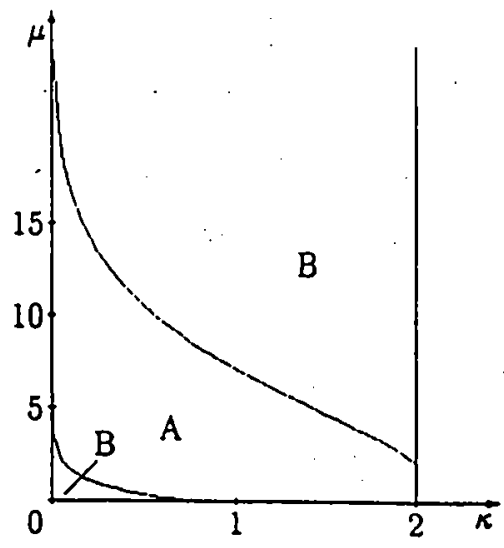

$A$... optimal according to Remark 5.2

$A \cup B \ldots$ order optimal according to Remark 5.3 


\section{Optimality of iterated Tikhonov methods}

In these regularization methods $x_{a}^{\delta}$ is obtained after performing $m$ steps of the generalized method of Tikhonov regularization as discussed in Section 3, i.e. there holds $x_{\alpha}^{\delta}:=x_{m}$, where $x_{m}$ is obtained according to

$$
\begin{aligned}
x_{0} & =\bar{x} \\
x_{k+1} & =x_{k}+\left[\left(A^{*} A\right)^{s+1}+\alpha I\right]^{-1}\left(A^{*} A\right)^{s} A^{*}\left[y^{\delta}-A x_{k}\right] \quad(k=0,1, \ldots, m-1) .
\end{aligned}
$$

Consequently, $x_{\alpha}^{\delta}$ can be represented in the form (1.4) with $g_{\alpha}(t)=\left[1-\left(\frac{\alpha}{(\alpha+t)}\right)^{m}\right] / t$. For the functions $g$ and $h$ of $(2.1)$ there holds $g(t)=\left[1-\left(\frac{1}{1+t}\right)^{m}\right] / t$ and $h(t)=\left(\frac{1}{1+t}\right)^{m}$. The equation $h(t)=1 /(p+1)$ has the unique solution $t_{0}=(p+1)^{1 / m}-1$, hence, from Theorem 2.3 we obtain

Corollary 6.1. Let $A \in \mathcal{L}(X, Y)$ and $x_{\alpha}^{\delta}:=x_{m}$ the regularized solution of method (6.1) where $\alpha$ is given by the a priori parameter choice $\alpha=\frac{1}{(p+1)^{1 / m}-1}\left(\frac{\delta}{E}\right)^{(2 s+2) /(p+1)}$. If the constants $\kappa$ and $\mu$ from (2.4) satisfy the inequality $\sup _{y \geq 0} f(y) \leq 1$ with

$$
\begin{aligned}
f(y)= & \frac{\kappa+\mu}{\mu}\left[\left(\frac{\kappa+\mu}{\kappa}\right)^{1 / m}-1\right]^{-\mu} y^{\mu}(1+y)^{-2 m} \\
& +\frac{\kappa+\mu}{\mu}\left[\left(\frac{\kappa+\mu}{\kappa}\right)^{1 / m}-1\right]^{\kappa} y^{-\kappa}\left[1-(1+y)^{-m}\right]^{2},
\end{aligned}
$$

then there holds the error estimate (3.3).

Remark 6.2. In order to check for which $(\kappa, \mu)$-values the method (6.1) is optimal on $M_{p, E}$ we have examined the function $f(y)$ of Corollary 6.1 numerically for different integers $m$. Figures 1 and 4 show the corresponding $(\kappa, \mu)$-ranges for which $\sup _{y \geq 0} f(y) \leq 1$ in the cases $m=1$ and $m=2$.

The next theorem shows that for growing $m$ the $(\kappa, \mu)$-range for which the method (6.1) is optimal tends to the $(\kappa, \mu)$-range for which the method (5.1) is optimal.

Theorem 6.3. Let $A \in \mathcal{L}(X, Y), \kappa$ and $\mu$ given by (2.4) and $\alpha=\frac{1}{t_{0}}\left(\frac{\delta}{E}\right)^{2 /(\kappa+\mu)}$ with $t_{0}=\left(\frac{\kappa+\mu}{\kappa}\right)^{1 / m}-1$. Then the method (6.1) is for $m \rightarrow \infty$ optimal on the set $M_{p, E}$ if $\sup _{y \geq 0} f(y) \leq 1$ holds where $f(y)$ is the function from Corollary 5.1.

Proof. We use Lemma 2.1 with $g_{\alpha}(t)=\left[1-\left(\frac{\alpha}{\alpha+\imath}\right)^{m}\right] / t$, substitute $t^{s+1}=z$ and obtain with the notations (2.4) and the choice $\xi=\kappa /(\kappa+\mu)$ that

$$
\begin{aligned}
& \Delta(\delta)=\inf _{0<\xi<1} \sup _{t \in \sigma\left(A^{*} A\right)}\left\{\frac{E^{2}}{\xi} t^{p}\left[1-t^{s+1} g_{\alpha}\left(t^{s+1}\right)\right]^{2}+\frac{\delta^{2}}{1-\xi} t^{2 s+1} g_{\alpha}^{2}\left(t^{s+1}\right)\right\}^{1 / 2} \\
& \leq \sup _{z \geq 0}\left\{\frac{\kappa+\mu}{\kappa} E^{2} z^{\mu}\left[1-z g_{\alpha}(z)\right]^{2}+\frac{\kappa+\mu}{\mu} \delta^{2} z^{2-\kappa} g_{\alpha}^{2}(z)\right\}^{1 / 2} .
\end{aligned}
$$


Note that

$$
\lim _{m \rightarrow \infty} g_{\alpha}(z)=\frac{\left[1-e^{-z / \alpha_{1}}\right]}{z} \quad \text { with } \quad \alpha_{1}=\frac{1}{\ln \left(\frac{\kappa+\mu}{\kappa}\right)}\left(\frac{\delta}{E}\right)^{2 /(\kappa+\mu)} \text {, }
$$

consequently,

$$
\lim _{m \rightarrow \infty} \Delta(\delta) \leq \sup _{x \geq 0}\left\{\frac{\kappa+\mu}{\kappa} E^{2} z^{\mu} e^{-2 z / \alpha_{1}}+\frac{\kappa+\mu}{\mu} \delta^{2} z^{-\kappa}\left[1-e^{-z / \alpha_{1}}\right]^{2}\right\}^{1 / 2}
$$

We substitute $z=\alpha_{1} y$ and obtain

$$
\begin{aligned}
\lim _{m \rightarrow \infty} \Delta(\delta) & \leq \sup _{y \geq 0}\left\{\frac{\kappa+\mu}{\kappa} E^{2}\left(\alpha_{1} y\right)^{\mu} e^{-2 y}+\frac{\kappa+\mu}{\mu} \delta^{2}\left(\alpha_{1} y\right)^{-\kappa}\left[1-e^{-y}\right]^{2}\right\}^{1 / 2} \\
& =E^{\frac{\kappa}{\kappa+\mu}} \delta^{\frac{\mu}{\kappa+\mu}} \sup _{y \geq 0}\{f(y)\}^{1 / 2}
\end{aligned}
$$

where $f(y)$ is the function from Corollary 5.1. This completes the proof

Remark 6.4. Let $(\kappa, \mu) \in(0,2] \times(0,2 m]$. Then the method (6.1) is order optimal on $M_{p, E}$ for the parameter choice $\alpha=k\left(\frac{\delta}{E}\right)^{(2 s+2) /(p+1)}$ with a constant $k>0$. The proof of this result follows from Remark 2.5. The $(\kappa, \mu)$-range in which the method (6.1) is optimal or order optimal on $M_{p, E}$ (for $m=2$ ) is given in Figure 4.

Figure 4. $(\kappa, \mu)$-range of optimality for method (6.1) with $m=2$

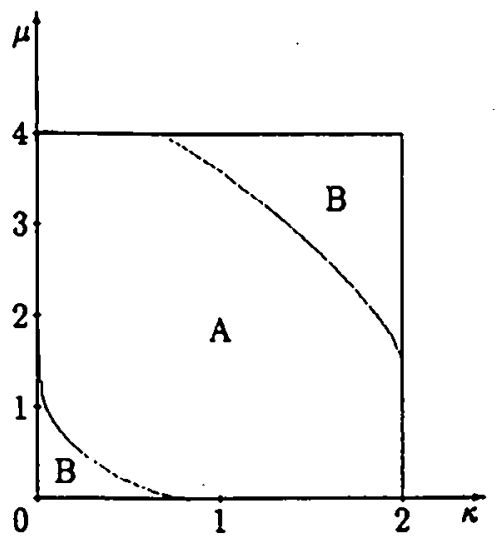

$A$... optimal according to Remark 6.2

$A \cup B$... order optimal according to Remark 6.4 


\section{Asymptotical optimality of Landweber iterations}

In this section we examine the generalized Landweber iteration as a special representative of more general iteration methods (cf., e.g., [3]). In these regularization methods the regularized solution $x_{N}^{\delta}$ is determined by

$$
x_{k+1}^{\delta}=x_{k}^{\delta}-\left(A^{*} A\right)^{s} A^{*}\left(A x_{k}^{\delta}-z^{\delta}\right) \quad\left(k=0,1, \ldots, N-1 ; x_{0}^{\delta}=\bar{x}\right),
$$

hence, $x_{N}^{\delta}$ has the representation (1.4) with $g_{\alpha}(t)=\left[1-(1-t)^{N}\right] / t$ and $\alpha=1 / N$ is the regularization parameter. Since the regularization parameter $\alpha=1 / N$ takes only discrete values it is impossible to discuss optimality results of the method (7.1), hence we have to switch over to the concept of asymptotical optimality. The next theorem shows that the generalized Landweber iteration (7.1) is asymptotically optimal for such $(\kappa, \mu)$-values, for which the generalized method of asymptotical regularization $(5.1)$ is optimal. The proof of this result is based on a corresponding result for the special case $s=0$ which can be found in [14].

Theorem 7.1. Let $A \in \mathcal{L}(X, Y)$ with $\left\|\left(A^{*} A\right)^{s+1}\right\| \leq 2, \kappa$ and $\mu$ given by (2.4) and $N=\operatorname{int}\left\{\ln \left(\frac{\kappa+\mu}{\kappa}\right)\left(\frac{\delta}{E}\right)^{-2 /(\kappa+\mu)}\right\}$ (int $\{\lambda\}$ denotes the integer part of the real number $\lambda$ ). Then the method (7.1) is asymptotically optimal on the set $M_{p, E}$ if $\sup _{y \geq 0} f(y) \leq 1$ holds, where $f(y)$ is the function from Corollary 5.1 .

Proof. We use (6.2) with $g_{\alpha}(t)=\left[1-(1-t)^{N}\right] / t$, substitute $t^{s+1}=z$ and obtain with the notations (2.4) and the choice $\xi=\kappa /(\kappa+\mu)$ that

$$
\begin{aligned}
\Delta(\delta) & =\inf _{0<\xi<1} \sup _{z \in \sigma\left(\left(A^{*} A\right)^{\circ+1}\right)}\left\{\frac{E^{2}}{\xi} z^{\mu}(1-z)^{2 N}+\frac{\delta^{2}}{1-\xi} z^{-\kappa}\left[1-(1-z)^{N}\right]^{2}\right\}^{1 / 2} \\
& \leq \sup _{0 \leq z \leq 2}\left\{\frac{\kappa+\mu}{\kappa} E^{2} z^{\mu}(1-z)^{2 N}+\frac{\kappa+\mu}{\mu} \delta^{2} z^{-\kappa}\left[1-(1-z)^{N}\right]^{2}\right\}^{1 / 2} \\
& \equiv \sup _{0 \leq z \leq 2} g(z)=\max \left\{s_{N}(\kappa, \mu, \delta, E), \sigma_{N}(\kappa, \mu, \delta ; E)\right\}
\end{aligned}
$$

holds, where $s_{N}=\sup _{0 \leq z \leq r / N} g(z)$ and $\sigma_{N}=\sup _{\tau / N \leq z \leq 2} g(z)$. In analogy to the proof of Theorem 6.2 in [14] it can be shown that for some suitable chosen $\tau>0$ there holds

(i) $\sigma_{N}^{2} \leq E^{2 \kappa /(\kappa+\mu)} \delta^{2 \mu /(\kappa+\mu)}$

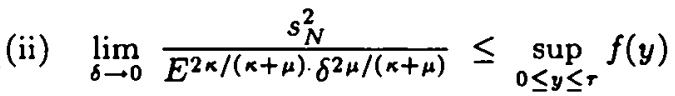

where $f(y)$ is the function from Corollary 5.1. From (i) and (ii) we obtain the statement 
Remark 7.2. Let $(\kappa, \mu) \in(0,2] \times(0, \infty)$. Then the generalized Landweber iteration (7.1) is order optimal on $M_{p, E}$ for the parameter choice $N=\operatorname{int}\left\{k\left(\frac{\delta}{E}\right)^{-2 /(\kappa+\mu)}\right\}$ with a constant $k>0$. The proof of this result follows from (7.2) with $\xi=1 / 2$ and from the two inequalities

(i) $z^{\mu}(1-z)^{2 N} \leq z^{\mu} e^{-2 N z} \leq N^{-\mu}\left(\frac{\mu}{2}\right)^{\mu} e^{-\mu}$

(ii) $z^{-\kappa}\left[1-(1-z)^{N}\right]^{2} \leq N^{\kappa}$

thiat hold for all $z \in[0,2]$ and $(\kappa, \mu) \in(0,2] \times(0, \infty)$.

\section{References}

[1] Baumeister, J. : Stable Solution of Inverse Problems. Braunschweig: Vieweg 1987.

[2] Engl, H. W. and H. Gfrerer: A posteriori parameter choice for general regularization methods for solving linear ill-posed problems. Appl. Numer. Math. 4 (1988), $395-417$.

[3] Hanke, M. : Accelerated Landweber iterations for the solution of ill-posed equations. Numer. Math. 60 (1991), 341 - 373.

[4] Hofmann, B. : Regularization for Applied Inverse and Ill-Posed Problems (Teubner - Texte zur Mathematik: Vol. 85). Leipzig: B. G. Teubner Verlagsges. 1986.

[5] Kiho, T. : Optimal choice of the parameter in the iterated versions of the Tikhonov method on the source classes of solutions (in Russian). Preprint. Tartu: University of Tartu, Preprint 863, 1989, pp. 95 - 99. In Russian.

[6] Louis, A. K. : Inverse und schlecht gestellte Probleme. Stuttgart: B. G. Teubner 1989.

[7] Melkmann, A. A. and C. A. Micchelli: Optimal estimation of linear operators in Hilbert spaces from inaccurate data. SIAM J. Numer. Anal. 16 (1979), 87 - 105.

[8] Natterer, F.: Error bounds for Tikhonov regularization in Hilbert scales. Applic. Analysis 18 (1984), $29-37$.

[9] Seidmann, T. I. : 'Optimal Filtering' for some ill-posed problems. In : Wave Propagation and Inversion (eds.: W. E. Fitzgibbon and M. F. Wheler). Philadelphia (PA, USA): SIAM 1992, pp. $108-123$.

[10] Schröter, T. and U. Tautenhahn: Error estimates for Tikhonov regularization in Hilbert scales. Numer. Funct. Anal. and Optimiz. 15 (1994), $155-168$.

[11] Tautenhahn, U. : Error estimates for regularization methods in Hilbert scales. Preprint. Chemnitz: Techn. Univ. Chemnitz-Zwickau, Preprint 240, 1993.

[12] Tikhonov, A. N. and V. Y. Arsenin: Solution of Ill-Posed Problems. New York: Wiley 1977.

[13] Vainikko, G. M. and A. Y. Veretennikov: Iteration Procedures in Ill-Posed Problems (in Russian). Moscow: Nauka 1986.

[14] Vainikko, G. M. : On the optimality of methods for ill-posed problems, Z. Anal. Anw. Bd. 6 (1987), $351-362$.

[15] Vainikko, G. M. : On the optimality of regularization methods. In: Inverse and Ill-Posed Problems (eds.: H. W. Engl and W. Groetsch). Orlando: Acad. Press 1987, pp. $77-95$. 